\title{
A CELLULAR AUTOMATON MODEL FOR A PEDESTRIAN FLOW PROBLEM*
}

\author{
Jiří FelCMAN ${ }^{1, * *}$ AND Petr KuBERA Ken $^{2,3}$
}

\begin{abstract}
The evacuation phenomena in the two dimensional pedestrian flow model are simulated. The intended direction of the escape of pedestrians in panic situations is governed by the Eikonal equation of the pedestrian flow model. A new two-dimensional Cellular Automaton (CA) model is proposed for the simulation of the pedestrian flow. The solution of the Eikonal equation is used to define the probability matrix whose elements express the probability of a pedestrian moving in finite set of directions. The novelty of this paper lies in the construction of the density dependent probability matrix. The relevant evacuation scenarios are numerically solved. Predictions of the evacuation behavior of pedestrians, for various room geometries with multiple exits, are demonstrated. The mathematical model is numerically justified by comparison of CA approach with the Finite Volume Method for the space discretization and Discontinuous Galerkin Method for the implicit time discretization of pedestrian flow model.
\end{abstract}

Mathematics Subject Classification. 76N15, 70B05.

Received June 30, 2020. Accepted December 25, 2020.

\section{INTRODUCTION}

The increase in the number of people living in cities represents a higher demand on modern urban modeling and planning. The large accumulation of people brings serious problems in panic situations, such as, floods, fires, crowd psychosis during services, or sports events, and concerts. In such cases, it is necessary to have evacuation plans in place and try to predict people's behavior. The Pedestrian and Evacuation Dynamics (PED) is an interesting non-linear complex system where, as well as the physical and environmental conditions (e.g. visibility, see [21]), we must also account for the sociological and psychological conditions (e.g. fatigue, see [13]). The complexity of the pedestrian behaviour can be seen in the collective effects and self-organisation phenomena.

The description of PED can be given by a mathematical model. PED models can be classified according to the scale of the described phenomena, as macroscopic, mesoscopic or microscopic. In the following paragraphs

\footnotetext{
* The research was supported by the grant No. UJEP-IGA-TC-2019-53-02-2 of IGA UJEP. The authors acknowledge this support.

Keywords and phrases: Pedestrian flow, cellular atomaton, Eikonal equation, finite volume method, fast sweeping method.

${ }^{1}$ Department of Numerical Mathematics, Faculty of Mathematics and Physics, Charles University, Prague, Czech Republic.

2 Department of Informatics, Faculty of Nuclear Sciences and Physical Engineering, Czech Technical University, Prague, Czech Republic.

${ }^{3}$ Department of Software Engineering, Faculty of Science, Jan Evengelista Purkyně University, Ústí nad Labem, Czech Republic.

** Corresponding author: felcman@karlin.mff.cuni.cz
} 
we briefly describe those classifications; more details about a particular model and its details can be found in $[6]$.

From the macroscopic point of view the studied PED object represents a crowd and Computational Fluid Dynamics (CFD) methods are used, see e.g. [2, 27]. The macroscopic model is described by partial differential equations. See Section 2 for a brief introduction of a macroscopic model.

At the mesoscopic scale the system is described by an appropriate probability distribution over the microscopic states of the interacting particles, see e.g. kinetic models described in [8].

For the microscopic scale, the examined object represents an individual pedestrian (particle) and the crowd is modelled as a multi-particle system. According to the used modelling formalism, microscopic models can be divided into several groups. The first group includes models where the pedestrian movement is described by ordinary differential equations. The fundamental paper in this area [15], considers a social force model where the pedestrian movement is driven by his motivation. Another social force model, where the desired direction of the pedestrian motion is given by the solution of the Eikonal equation, can be found in [16]. A further example of ordinary differential equation formalism is a gradient navigation model, where the pedestrian movement is driven by the gradient of the distance function from obstacles and other pedestrians, see e.g. [7].

The second group includes models where the pedestrian movement and their behaviour are described by a system of rules. The Agent-Based Model (ABM), see [24, 26], and CA models serve as examples of this group. For the detailed taxonomy of CA models see the overview in [20]. One of the most used CA model is the Floor Field Cellular Automata (FFCA) model, described in [5, 18]. Currently developed FFCA models can be found, e.g., in $[13,14,21]$. In this model the probability of pedestrian movement in some direction is determined by two floor fields: static and dynamic. The former field is static in time and is given by the geometry of the domain; while the later is used to model an attractive interaction between pedestrians and is dynamically modified by the presence of pedestrians. Further examples of CA models are the Cost Potential Field (CPF) models, see e.g. [29]. Here the cost potential represents the minimal cost for traveling from the current position to the exit. The probability of pedestrian movement in some direction is given by the gradient of the cost potential. The cost potential is also used in microscopic non-CA models [7, 16] and macroscopic models; see e.g. [17, 19, 27].

We have been interested in the solution of the pedestrian flow equations from the macroscopic point of view in several papers, namely in $[9,19]$. We have presented an efficient and robust algorithm for the numerical solution of the room evacuation problem. The aim was to perform numerical simulations and provide a time evolution of the pedestrian density distribution in the event of a room evacuation and to show trajectories of pedestrians. We have verified and compared the numerical results using the various numerical methods for the solution of the partial differential equations (splitting techniques, finite volume method with different numerical fluxes, discontinuous Galerkin method). This verification issues led us to the idea to compare directly the numerical results of two different worlds: macroscopic (PDE) and microscopic (CA) one.

Inspired by the principles of the FFCA model [5], the CPF model [29], and by the role of the Eikonal equation in the macroscopic approach [27], we propose in this paper a new cellular automaton as a microscopic model and compare the obtained numerical results with the macroscopic CFD approach for the pedestrian flow. It is important that the same data settings are used for both of these models. Our CA model uses an exponential type of probability dependency (similar to FFCA and different from CPF) on the cost potential given by the Eikonal equation (different from FFCA and similar to CPF). This is the novel result of this paper.

The macroscopic model we shall use for the comparison is the two-dimensional model derived in detail in [9]. For comparison purposes, we briefly remember its construction.

\section{MACROSCOPIC MODEL}

In the case of macroscopic models, pedestrians are considered as a continuum with their motion in the (polygonal) domain $\Omega \subset \mathbb{R}^{2}$ and time interval $(0, T)$ with $T>0$, described by the two-dimensional equations of fluid dynamics in terms of density of pedestrians $\rho$ in $\left[\mathrm{ped} / \mathrm{m}^{2}\right]$, by their velocity $\boldsymbol{v}=\left(v_{1}, v_{2}\right)$ in $[\mathrm{m} / \mathrm{s}]$ and by the pressure $p$ in $\left[\mathrm{ped} / s^{2}\right.$. These equations are derived from the law of conservation of mass and the law of conservation of momentum for the inviscid fluid. The equations are considered in the bounded domain $\Omega$ 
(stadium, concert hall, school classroom), whose boundary $\partial \Omega$ is formed by the walls $\Gamma_{w}$ and by the exits (outlets) $\Gamma_{o}, \partial \Omega=\bar{\Gamma}_{w} \cup \bar{\Gamma}_{o}$.

The governing equations are

$$
\frac{\partial \rho}{\partial t}+\operatorname{div}(\rho \boldsymbol{v})=0
$$

(conservation of mass, continuity equation), and

$$
\frac{\partial \rho \boldsymbol{v}}{\partial t}+\operatorname{div}(\rho \boldsymbol{v} \otimes \boldsymbol{v})=\rho \mathbf{f}-\nabla p
$$

(the conservation of momentum). These equations can be found in every fluid dynamics textbook, see e.g. [11]. The pressure $p$ is eliminated from the momentum equation (2.2) supposing the power law for isentropic gases

$$
p=\tilde{p}(\rho)=p_{0} \rho^{\gamma}, p_{0}>0, \gamma>1 .
$$

The physically relevant data are $p_{0} \in[0.0005,10]$ and $\gamma \in[2,5]$. These data are chosen from experimental studies of pedestrian behavior (see $[3,25]$ ).

The model is further completed by the specification of the term $\mathbf{f}$. The quantity $\mathbf{f}$ has the meaning of the outer volume forces. The model is based on the traffic models described in $[22,28]$ and it was proposed in [17]. The model and its various aspects are deeply studied in [27]. We refer to [2] for related representation problems. We follow the approach formulated in [27], where the term $\mathbf{f}$ with the dimension $\left[\mathrm{m} / \mathrm{s}^{2}\right]$ is defined as

$$
\mathbf{f}=\frac{1}{\tau}\left(\boldsymbol{v}_{p e d}-\boldsymbol{v}\right)
$$

The variable $\boldsymbol{v}_{p e d}$ represents the velocity at which a real pedestrian in a real situation will move at time instant $t$. The parameter $\tau$ is a relaxation time describing how fast pedestrians correct their current velocity $\boldsymbol{v}$ to the desired one $\boldsymbol{v}_{p e d}$ and is set, as $\tau=0.61$ according to the validation of the model with experimental pedestrian movement data. The quantity $\boldsymbol{v}_{\text {ped }}$ depends at time $t$ in the macroscopic setting on the distribution of the density $\rho(\cdot, t)$ in the whole domain $\Omega$. There is no closed formula for the determination of $\boldsymbol{v}_{\text {ped }}$, the evaluation of $\boldsymbol{v}_{\text {ped }}$ is based on the solution of the so called Eikonal equation of the pedestrian flow. Since the Eikonal eqation will be used also in the CA approach, we describe the determination of the $\boldsymbol{v}_{\text {ped }}$ simultaneously with the Eikonal equation in the next section.

\subsection{Eikonal equation of pedestrian flow}

The Eikonal equation plays a crucial role for the determination of the velocity $\boldsymbol{v}_{\text {ped }}$ in the macroscopic model momentum equation (2.2) and is a basis for the CA approach we propose. We briefly show the derivation of the Eikonal equation as a tool for the determination of the quantity $\boldsymbol{v}_{\text {ped }}$. The use of the Eikonal equation in the CA approach and the motivation will then be more clear. The details can be found in [9].

The macroscopic model from Section 2 is based on the assumption that the pedestrian at time $\bar{t}$ knows the density distribution $\rho(\cdot, \bar{t})$ in the whole $\Omega$. From this distribution, it determines the absolute value of the velocity $\left|\boldsymbol{v}_{\text {ped }}\right|$ in the dependence on the density $\rho$. The basic consideration is that in a place of high density the speed will be low (due to a large accumulation of people), while in a place of low density the speed will be high (small number of people). An empirical formula was established in [27]:

$$
\begin{aligned}
\left|\boldsymbol{v}_{p e d}\right| & =\widetilde{V}(\rho), \\
\widetilde{V}(\rho) & =v_{\max } e^{-\alpha\left(\frac{\rho}{\rho_{\max }}\right)^{2}} .
\end{aligned}
$$


For futher details see [4]. We see from formula (2.5) that if the density at point $x \in \Omega$ is close to zero, pedestrians will go at speed close to $v_{\max }$. The free flow speed $v_{\max } \in[1,7]$ is considered. In $(2.5)$ the maximum density $\rho_{\max }$ at which the motion is hardly possible is $\rho_{\max }=9$ (nine pedestrians per square meter) and $\alpha=7.5$. These data are chosen from the experimental investigation of pedestrian behavior. We have chosen relation (2.5) in order to have the same model as in [27] for comparison reasons.

Having denoted the dependence of the magnitude of the velocity $\boldsymbol{v}_{\text {ped }}$ on the density $\rho$ as $\widetilde{V}(\rho)$, the velocity $\boldsymbol{v}_{\text {ped }}$ can be expressed

$$
\boldsymbol{v}_{p e d}=\widetilde{V}(\rho) \boldsymbol{\mu},
$$

where $\boldsymbol{\mu}$ denotes the unit vector. The determination of the direction $\boldsymbol{\mu}$ is based on the consideration that the pedestrian attempts to start his walk at time $\bar{t}$ in a direction $\boldsymbol{\mu}$ such that he reaches the exit in the shortest possible time. This means that at time $\bar{t}$ the pedestrian chooses a path to the exit such that the travel time along this path is minimal. Then, the unit tangential vector to this path at the starting point is taken as $\boldsymbol{\mu}$. More precisely, let $\boldsymbol{\eta}(\sigma ; x)$ be the curve starting at $x$ and ending on the exit $\Gamma_{o}$, parametrized by the parameter $\sigma \in[0,1]$. Since for the straight line

$$
\text { time }=\frac{\text { length of the path }}{\text { speed }}
$$

the time $\tilde{T}$ to move along the curve $\boldsymbol{\eta}(\sigma ; x)$ can be defined by the curvilinear integral

$$
\tilde{T}[\boldsymbol{\eta}]=\int_{\boldsymbol{\eta}} \frac{\mathrm{d} S}{\widetilde{V}(\rho(\cdot, \bar{t}))} .
$$

(see [9] for details.) We come finally to the definition of $\boldsymbol{\mu}$. Let the density $\rho$ at the time $\bar{t}$ be given. We say that $\varphi$ is the fastest path from $x \in \Omega$ to the exit $\Gamma_{o}$ at the time $\bar{t}$, if

$$
\boldsymbol{\varphi}=\arg \min _{\boldsymbol{\eta}(\sigma ; x)} \tilde{T}[\boldsymbol{\eta}] .
$$

Here, the minimum is taken over all piecewise regular curves $\boldsymbol{\eta}$ in $\Omega$, parametrized by the parameter $\sigma \in[0,1]$ with the property $\boldsymbol{\eta}(0 ; x)=x, \boldsymbol{\eta}(1 ; x) \in \Gamma_{o}$ and $\tilde{T}[\boldsymbol{\eta}]$ given by relation (2.8). Hence the fastest path is defined by the shortest time needed to move along it. The desired direction of movement $\boldsymbol{\mu}$ is then given as the unit tangential vector at the point $x$ to the fastest path in $\Omega$ from $x$ to the exit $\Gamma_{o}$

$$
\boldsymbol{\mu}(x, \bar{t})=\frac{\varphi^{\prime}(0 ; x)}{\left|\varphi^{\prime}(0 ; x)\right|} .
$$

( $\varphi^{\prime}$ means the derivative with respect to the parameter $\sigma$.)

It was proved in [9] that $\boldsymbol{\mu}$ can be equivalently expressed as

$$
\boldsymbol{\mu}(x, t)=-\frac{\nabla \phi(x)}{|\nabla \phi(x)|}
$$

where the function $\phi(x)$ is the solution of the so called Eikonal equation of the pedestrian flow

$$
|\nabla \phi(x)|=\frac{1}{\widetilde{V}(\rho(x, \bar{t}))}, \quad x \in \Omega
$$



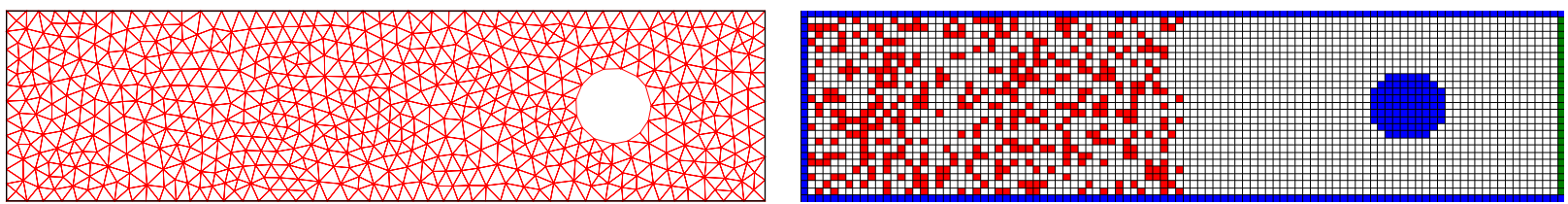

FiguRE 1. Rectangular domain with one circular obstacle at point $[32,5]$ with radius $R=2 \mathrm{~m}$. Triangulation for a macroscopic model (left), CA with cells in states empty, pedestrian, wall and outflow colored as white, red, blue and green, respectively (right).

equipped by the boundary condition

$$
\phi(x)=0, \quad x \in \Gamma_{o} .
$$

It was proved in [9], that the solution $\phi$ of the Eikonal equation (2.12) represents the time it takes for a pedestrian to reach an exit point in $\Gamma_{o}$ if the pedestrian follows the curve $\varphi$ satisfying relation $(2.9)$, i.e. $\phi(x)=\tilde{T}[\varphi]$, where $\tilde{T}$ is defined in $(2.8)$.

\subsection{Pedestrian flow eqations}

Summarizing the previous considerations, we can define the pedestrian flow problem as finding the density $\rho$ and the velocity $\boldsymbol{v}$ satisfying the continuity equation (2.1) and the momentum equation (2.2) completed by the Eikonal equation (2.12). The Eikonal equation plays an important role for choosing the direction in which the pedestrian will head towards the exit. In the momentum equation (2.2) the pressure $p$ is given by relation (2.3), the quantity $\mathbf{f}$ is defined in (2.4), the pedestrian correction velocity $\boldsymbol{v}_{\text {ped }}$ is expressed by (2.6), the intended pedestrian magnitude velocity $\widetilde{V}$ can be found in the empirical formula (2.5) and the intended direction of movement $\boldsymbol{\mu}$ of a pedestrian is defined by the solution $\phi$ of the Eikonal equation (2.12) by formula (2.11). In the functions under consideration, the space variable $x$ is considered in the closure of the computational domain $\Omega \subset \mathbb{R}^{2}$ with the boundary $\partial \Omega$ formed by the walls $\Gamma_{w}$ and by the exits (outlets) $\Gamma_{o}, \partial \Omega=\bar{\Gamma}_{w} \cup \bar{\Gamma}_{o}$. The time variable is considered in the interval $(0, T)$ with $T>0$. The system is equipped by the initial and boundary conditions. Since the boundary conditions are precisely specified usually at the discrete level of the solution, we refer at this point to [9], where the numerical solution procedure is described. As an example of the computational domain from [9], the triangulation of the rectangular domain $\Omega=(0,40) \times(0,10)$ with a circular obstacle at point $[32,5]$ with radius $R=2 \mathrm{~m}$ and the exit formed by the whole right side $\left\{x=\left(x_{1}, x_{2}\right), x_{1}=40, x_{2} \in(0,10)\right\}$ of the rectangle is depicted in Figure 1 (left).

\section{Microscopic MODEL}

In the microscopic model each pedestrian is considered individually and its position and velocity is simulated. This can be done e.g. via $N_{\text {ped }}$ ordinary differential equations, where $N_{\text {ped }}$ is the number of pedestrians. See [10] for a simulation of pedestrian behaviour in overcrowding places based on the microscopic approach. Here, we concentrate on the cellular automaton approach. The physical meaning of the solution of the Eikonal equation (2.12) inspired its use in the CA approach. Although macroscopic and microscopic models represent two different worlds, we show their comparison and interrelationship. We further enlarge the results from [12].

\subsection{Computational domain}

Let us consider the evacuation of pedestrians in the polygonal domain $\Omega \subset \mathbb{R}^{2}$ and time interval $(0, T)$ with $T>0$. We suppose that the boundary $\partial \Omega=\bar{\Gamma}_{w} \cup \bar{\Gamma}_{o}$ as defined in Section 2.2 .

In $\bar{\Omega}$ a mesh $\mathcal{T}_{h}$ of closed square cells $C$ is constructed, $\bar{\Omega}=\bigcup_{C \in \mathcal{T}_{h}} C$. The ghost cells are added to the mesh $\mathcal{T}_{h}$. Let us denote the set of ghost cells adjacent to the boundary $\Gamma_{w}$ and $\Gamma_{o}$ by $\mathcal{T}_{w}$ and $\mathcal{T}_{o}$, respectively. In 
Figure 1 (right) the mesh $\mathcal{T}_{h}$ for the rectangular domain $\Omega=(0,40) \mathrm{x}(0,10)$ with an obstacle and exit formed by the whole right side $\left\{x=\left(x_{1}, x_{2}\right), x_{1}=40, x_{2} \in(0,10)\right\}$ of the rectangle is depicted. The ghost cells $\mathcal{T}_{w}$ (walls and an obstacle) are in a blue color, and the ghost cells $\mathcal{T}_{o}$ (exit) are in green. We define the two-dimensional mesh $\mathcal{T}_{C A}=\mathcal{T}_{h} \cup \mathcal{T}_{w} \cup \mathcal{T}_{o}$, where each cell $C \in \mathcal{T}_{h}$ can either be empty or occupied by exactly one pedestrian. See Figure 1 (right), where the red cells represent the pedestrians. The size of an edge of a cell $C$ is $h=0.4 \mathrm{~m}$ which corresponds to a space occupied by a pedestrian in a dense crowd, see [5]. This is the simplest case which appears to be sufficient for most purposes.

On the mesh $\mathcal{T}_{h}$ the initial distribution of pedestrians is defined. See an example in Figure 1 (right), where pedestrians are denoted by red cells.

\subsection{Cellular automaton}

A CA approach for the pedestrian flow simulation is a method how to predict the evolution of the density distribution of pedestrians in time and, finally, the evacuation time. The simulation ends when there are no pedestrians in the room. The simulation starts at time $t=0$ for the given distribution of pedestrians (red cells) as for the example in Figure 1 (right). We select the time step $\tau$ such that we suppose that a single pedestrian moves with a velocity of one cell per time step, i.e. $0.4 \mathrm{~m}$ per time step. Empirically, the average velocity of a pedestrian is about $1.3 \mathrm{~m} / \mathrm{s}$ (see [5]). This gives a value of the time step $\tau=0.4 / 1.3=0.3 \mathrm{~s}$. The goal of the simulation is to provide the pedestrian density distribution at time instants $t_{k}=k \tau, k=0, \ldots$ During one time step the pedestrians change their positions according to the rules described below. This leads to the definition of a 2-dimensional cellular automaton, according to [1], as a structure $\mathcal{A}=\left(\mathbb{Z}^{2}, S, N, \delta\right)$ where $\mathbb{Z}^{2}$ is a discrete lattice of tuples of integers numbers, $S$ is a finite set of states, a neighborhood $N$ is a finite ordered subset of $\mathbb{Z}^{2}$ and $\delta: S^{m} \rightarrow S$ is a local transition function. Here $m$ stands for the number of neighbours of each cell.

We adapt the general definition of a $\mathrm{CA}$ for the particular pedestrian evacuation problem. In the following we identify the mesh $\mathcal{T}_{C A}$ with the certain finite lattice of a cellular automaton. The set $S$ is defined as four states $S=\{$ empty, pedestrian, wall, outflow $\}$ with corresponding colours $\{$ white, red, blue, green $\}$. We say that a cell $C \in \mathcal{T}_{C A}$ is in a empty (white) or pedestrian (red) state, if it is empty or occupied by a pedestrian, respectively. Wall (blue) and outflow (green) states are used to express boundary conditions on $\mathcal{T}_{w}$ and $\mathcal{T}_{o}$. As a neighborhood $N$ we consider the Moore neighborhood of the cell $N(C)$; see Figure 2 (left).

The transition function $\delta$ has to be specified for the CA approach. Since we are interested in the motion of pedestrians, we simulate it by the change of the density distribution, i.e. formally red cells are moving. The movement of pedestrians in the context of the CA is reached via changing the state of cells. To this end the local transition function $\delta^{*}$ defined for cells from $\mathcal{T}_{C A}$ with values in the set $S$ is introduced. For simplicity, we shall work with $\delta^{*}$ instead of a general fuction $\delta$ which involves a more complex definition of the notion of "state". In our approach, there is no explicit definition of $\delta^{*}$. We consider $\delta^{*}$ as set of rules based on which red cells change their position. Generally, in one time step, we accept nearest neighbour moves only. This means that the CA generates for each cell $C \in \mathcal{T}_{h}$ the direction of the move as indexes $(\mathrm{i}, \mathrm{j})$ indicating that the pedestrian goes from the cell $C=C_{22}$ to the cell $C_{i j} \in N(C)$. Here, we use the local notation from Figure 2 (left). First, we need to determine the direction of move. This will be described in the next section.

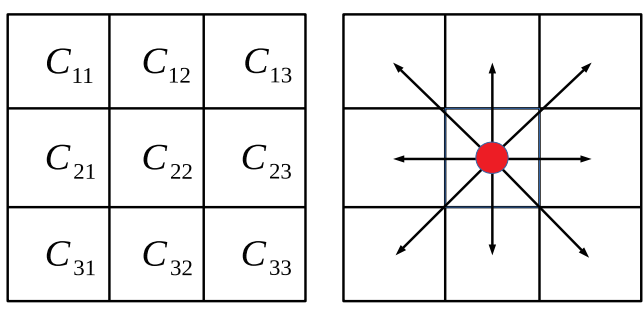

Figure 2. Moore neighborhood $N(C)$ of the cell $C$ formed locally by cells $C_{i j}, i, j=1, \ldots 3, C_{22}=C$ (left). Possible directions of move (right). 


\subsubsection{Desired direction of move}

Determining that a pedestrian will go from the cell $C \in \mathcal{T}_{h}$ to the cell $C_{i j} \in N(C), i, j \in\{1,2,3\}$ is based on the definition of the matrix $P^{C}=\left(p_{i j}^{C}\right)_{i, j=1}^{3}$ of probabilities that a pedestrian will move from $C \in \mathcal{T}_{h}$ to $C_{i j} \in \mathcal{T}_{C A}$. To each cell $C \in \mathcal{T}_{h}$ occupied by a pedestrian a $3 \times 3$ transition probability matrix $P^{C}=\left(p_{i j}^{C}\right)_{i, j=1}^{3}$ is assigned. This matrix is identified with the Moore neighborhood of the cell $C \in \mathcal{T}_{h}$ in such a way that the cell $C$ corresponds to the element $p_{22}^{C}$. The element $p_{i j}^{C}$ expresses the probability that a pedestrian at the cell $C \in \mathcal{T}_{h}$ will move to the target cell $C_{i j} \in \mathcal{T}_{C A}$ and $\sum_{i, j=1}^{3} p_{i j}^{C}=1$. The matrix $P^{C}$ is stored by rows forming a vector $P_{v e c}^{C}=\left(p_{11}^{C}, \ldots p_{33}^{C}\right)$. The interval $I=[0,1)$ is divided into 9 left half-closed subintervals $\left(I_{i j}\right)_{i, j=1}^{3}$ of lengths $p_{i j}^{C}$. A random number $r \in[0,1)$ is generated. The direction of move from $C \in \mathcal{T}_{h}$ to $C_{i j} \in \mathcal{T}_{C A}$ is defined in such a way that the indexes $i, j$ satisfy the relation $I_{i j} \ni r$. In order to satisfy the impermeability condition on the boundary $\Gamma_{w}$, all elements $p_{i j}^{C}$ which correspond to the $C_{i j} \in \mathcal{T}_{w}$ are set to zero. The construction of this matrix $P^{C}$ is described in Section 3.2.3.

The consequence of the mass conservation law is the necessity to solve conflicts in desired direction of movement. If two pedestrians occupy different cells $C^{(1)}, C^{(2)}$ and have the same empty target cell $C_{i j}^{(1)}=C_{k l}^{(2)}$, the realization of both transitions would lead to the situation that before the transition both cells were in the state pedestrian, while after the transition only one cell is in the new state pedestrian. To avoid this, we use similar rule as in [5]. The algorithm is described in the next section.

\subsubsection{Solving conflicts}

If two pedestrians occupy different cells $C^{(1)}, C^{(2)}$, have the same empty target cell $C_{i j}^{(1)}=C_{k l}^{(2)}$ and $p_{i j}^{(1)}>p_{k l}^{(2)}$ holds for the corresponding probabilities, the pedestrian at $C^{(1)}$ moves while the pedestrian at $C^{(2)}$ keeps his current position. This is the case when only $C^{(1)}$ and $C^{(2)}$ are in conflict. We proceed analogically for multiple conflicts. The highest probability authorizes the move and gives the highest priority. Since the conflicts could lead to chains of dependence, we use the above mentioned rule repeatedly; see Algorithm 1.

Algorithm 1: Changing states and solving conflicts.

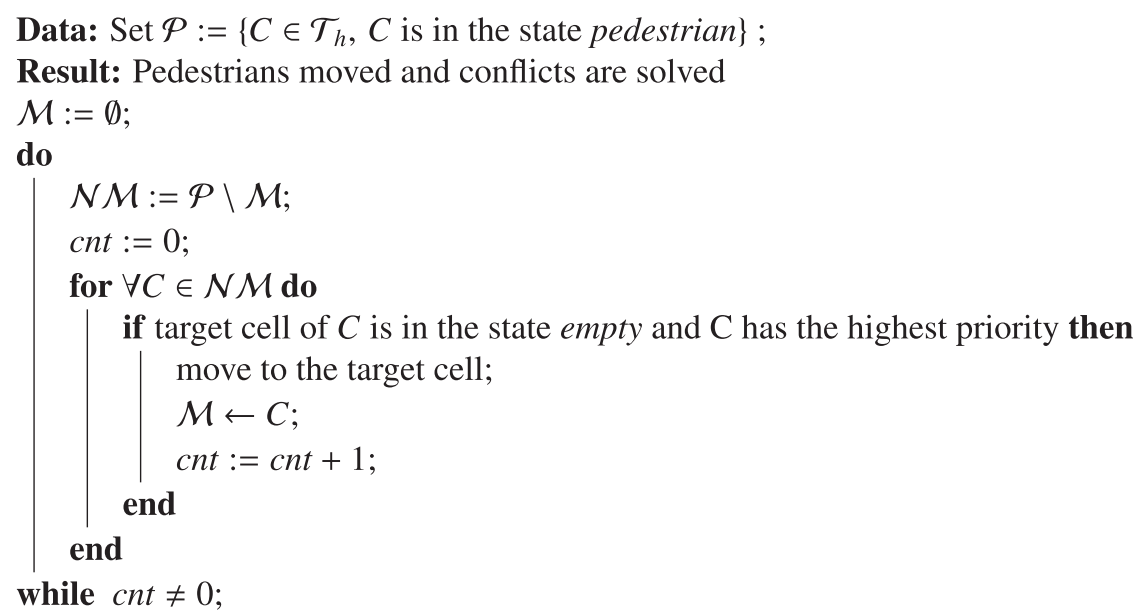

\subsubsection{Probability matrix $P^{C}$}

The probability that a pedestrian in the cell $C \in \mathcal{T}_{h}$ will move to the target cell $C_{i j} \in \mathcal{T}_{C A}$ is given by the intention of the pedestrian to reach the exit as soon as possible. We have mentioned in Section 2.1 that the solution $\phi(x)$ of the Eikonal equation of pedestrian flow represents the shortest time for the pedestrian at the position $x$ to reach the exit. This led us to the idea to define the probability that the pedestrian will move from 
$C \in \mathcal{T}_{h}$ to $C_{i j} \in \mathcal{T}_{C A}$ related to the time needed for the pedestrian to reach the exit from the cell $C_{i j} \in \mathcal{T}_{C A}$ : The longer the time to reach the exit is, the smaller the probability becomes. In our approach, the definition of the probability matrix $P^{C}$ is based on the piecewise constant approximation of the solution $\phi$ of the Eikonal equation (2.12), which is the novelty of this paper. Let us describe briefly the Godunov upwind difference scheme [30] which we use for the numerical solution of the Eikonal equation.

\subsubsection{Numerical solution of the Eikonal equation}

The piecewise constant approximation $\phi_{C}, C \in \mathcal{T}_{h}$ of the solution $\phi$ of equation (2.12) equipped by the boundary condition (2.13) is computed for each time instant $t_{k}, k=0,1, \ldots$. We approximate the restriction $\widetilde{V}\left(\left.\rho\left(\cdot, t_{k}\right)\right|_{C}\right), C \in \mathcal{T}_{h}$ of the velocity $\widetilde{V}(\rho)$ at the right hand side of $(2.12)$ by a constant $\widetilde{V}_{C}$ using formula $(2.5)$. In formula (2.5) the density $\rho$ is involved. In the CA approach we handle the density as follows.

With the pedestrian distribution at the time $t_{k}$ on $\mathcal{T}_{h}$ (see Figure 1 for the time instant $t_{0}$ ), we compute the constants $\rho_{C}=\# \operatorname{Ped} N(C) /|N(C)|, C \in \mathcal{T}_{h}$. Here \#PedN(C) is the number of pedestrians in the Moore neighborhood $N(C)$ of $C$ and $|N(C)|=1.44 \mathrm{~m}^{2}$ is the area of the Moore neighborhood of $C$. We fix $\rho_{C}=0$ for $C \in \mathcal{T}_{w} \cup \mathcal{T}_{o}$. Then, we approximate $\left.\rho\left(\cdot, t_{k}\right)\right|_{C}$ at the right hand side of $(2.12)$ by a constant $\rho_{C}$. So $\widetilde{V}_{C}=$ $\widetilde{V}\left(\rho_{C}\right), C \in \mathcal{T}_{C A}$ is well defined. Further, according to the boundary condition (2.13), we fix values $\phi_{C}=0$ for $C \in \mathcal{T}_{o}$.

We set the initial values $\phi_{C}=\phi_{\max }, C \in \mathcal{T}_{C A} \backslash \mathcal{T}_{o}$, where $\phi_{\max }$ is a large positive number $\left(\phi_{\max }=10^{5}\right)$ and use the following Godunov upwind difference scheme [30] to discretize the Eikonal equation (2.12)

$$
\left[\left(\phi_{C}-\min \left(\phi_{21}, \phi_{23}\right)\right)^{+}\right]^{2}+\left[\left(\phi_{C}-\min \left(\phi_{12}, \phi_{32}\right)\right)^{+}\right]^{2}=\frac{1}{\widetilde{V}_{C}^{2}} h^{2}, C \in \mathcal{T}_{h}
$$

We use the local notation of the Moore neighborhood $N(C), C \in \mathcal{T}_{h}$ (see Fig. 2) and denote $\phi_{i j}=\phi_{C_{i j}}$ for the approximation of the restriction $\phi$ on $C_{i j} \in \mathcal{T}_{C A}$. Furthermore, we set $(c)^{+}=\max (0, c)$.

For the solution of system (3.1) the Gauss-Seidel iterations with alternating orderings are proposed in [30]. For the given pedestrian density distribution $\rho_{C}$ at time instant $t_{k}$ the approximate solution $\phi_{C}, C \in \mathcal{T}_{h}$ is computed as follows.

We set for $C \in \mathcal{T}_{h} \phi_{C}^{\text {old }}:=\phi_{C}$ and compute the unique solution $\phi_{C}$ of (3.1) from the current values of its neighbors $\phi_{21}, \phi_{23}, \phi_{12}, \phi_{32}$ and the value $\widetilde{V}_{C}$. Then, the update $\phi_{C}:=\min \left(\phi_{C}^{\text {old }}, \phi_{C}\right)$ is defined. We sweep the mesh $\mathcal{T}_{h}$ with four alternating orderings repeatedly. We go through all the cells $C \in \mathcal{T}_{h}$ from left to right, downward and upward, then the same from right to left. Altogether we have four orderings. For the stopping criterion see [30].

\subsubsection{Elements of the matrix $P$}

Having computed the piecewise constant approximation of the solution $\phi$ of the Eikonal equation (2.12) at cells, we identify each Moore neighborhood of the cell $C \in \mathcal{T}_{h}$ with the matrix $P^{C}=\left(p_{i j}^{C}\right)_{i, j=1}^{3}$ in such a way that to the cell $C_{i j}$ (see Fig. 2) the transition probability $p_{i j}$ for the move from $C$ to $C_{i j}$ is assigned. The matrix $P^{C}$ is given by the local distribution of $\phi$ at cells of the Moore neighborhood of the cell $C \in \mathcal{T}_{h}$

$$
p_{i j}^{C}= \begin{cases}\tilde{N} e^{-\beta\left(\phi_{C_{i j}}-\phi_{C_{a v g}}\right)}, & \text { if } \phi_{C_{i j}}<\phi_{\max }, \\ 0, & \text { if } \phi_{C_{i j}}=\phi_{\max }\end{cases}
$$

where $\tilde{N}$ is the normalization factor to ensure $\sum_{i, j=1}^{3} p_{i j}^{C}=1, \phi_{C_{i j}}$ is the approximate value of the function $\phi$ at the cell $C_{i j}$ from the Moore neighborhood $N(C)$ of the cell $C \in \mathcal{T}_{h}, \phi_{C_{\text {avg }}}$ is the arithmetic average of 
piecewise constant approximate values of the potential $\phi$ at the Moore neighborhood $N(C)$ and $\phi_{\max }$ is a given large positive number $\left(\phi_{\max }:=10^{5}\right)$. In our numerical experiments we have taken $\beta \in(0,25)$. The parameter $\beta$ plays the similar role as the parameter $\alpha$ in (2.5) and is set experimentally. See numerical examples below for the setting of the parameter $\beta$. The exponential function in (3.2) was proposed to reflect the relationship of $p_{i j}^{C}$ to $\phi_{i j}$ mentioned in Section 3.2.3. We set $p_{i j}^{C}=0$ for each cell $C_{i j} \in N(C)$, for which $\phi^{C_{i j}}=\phi_{\max }$ and thus the movement of the pedestrian from $C$ to $C_{i j}$ is forbidden. Let us note that such situation occurs if $C_{i j} \in \mathcal{T}_{w}$.

\section{Cellular automaton flow chart}

Following the general strategy from [5], starting from the initial distribution of pedestrians (see e.g. Fig. 1 $\left(\right.$ right)), in each time step $t_{k}, k=1, \ldots$, for each pedestrian a desired direction of movement (see Sect. 3.2.1) to one of the cell from the Moore neighborhood is chosen. See Figure 2 (right) for the 8 possible directions of movement. Each pedestrian moves in the desired direction to one of the Moore neighborhood cell under the following conditions: If the target cell is occupied, the pedestrian does not move. If it is not occupied and no other pedestrian targets the same cell, the move is executed. If more then one pedestrians share the same target cell, one is chosen according to the rules described Section 3.2.2. The process is repeated in the next time step until the domain $\Omega$ is empty, i.e. no cell $C \in \mathcal{T}_{h} \cup \mathcal{T}_{o}$ is in the state pedestrian. The flow chart is demonstrated by Algorithm 2.

Algorithm 2: Evacuation simulation algorithm.

Data: All cells $C \in \mathcal{T}_{h}$ are set according to the initial condition, cells $C \in \mathcal{T}_{w}$ are set to the state wall and cells $C \in \mathcal{T}_{o}$ are set to the state outflow

Result: All cells $C \in \mathcal{T}_{h}$ are in state empty, states of cells from $\mathcal{T}_{w} \cup \mathcal{T}_{o}$ are unchanged

$t_{k}:=0$ (initial value of time);

while All cells $C \in \mathcal{T}_{h}$ are not in the state empty do

$\mathcal{P}:=\left\{C \in \mathcal{T}_{h}, C\right.$ is in the state pedestrian $\}$

$\forall C \in \mathcal{P}$ construct the probability matrix $P$ of the transition (see Section 2.2.3);

$\forall C \in \mathcal{P}$ determine the desired direction of move (see Section 2.2.1);

$\forall C \in \mathcal{P}$ solve conflicts in positioning and change states (see Section 2.2.2);

$t_{k}:=t_{k}+\tau$ (update time);

output pedestrian distribution at time $t_{k}$;

end

\section{NumericAl EXAMPLES}

The aim of this paper is to show how the proposed pedestrian Cellular Automaton is related to the Pedestrian Flow Equations described in Section 2.2 and to present a comparative study from a numerical point of view. Let us start with the evacuation of the rectangular domain $40 \times 10 \mathrm{~m}$ with one circular obstacle, see Figure 1. We suppose the outflow $\Gamma_{o}$ on the whole vertical right hand side and the wall boundary $\Gamma_{w}$ elsewhere. From the macroscopic point of view we use the following initial condition:

$$
\rho^{0}=\left\{\begin{array}{ll}
2 & \boldsymbol{x} \in[0,20] \times[0,10], \\
0 & \text { elsewhere }
\end{array} \text { and } \boldsymbol{v}^{0}=\mathbf{0} .\right.
$$

The setting of the initial condition $\rho^{0}$ corresponds to the continuous problem investigated in [9]. The CA initial condition is given by the pedestrian distribution red cells in Figure 1 (right). Let us note that for the given macroscopic initial density $\rho^{0}$ defined in (5.1) there are 400 pedestrians in the closure of the domain $\Omega_{P E D}:=(0,20) \times(0,10)$ at time $t_{0}$. This means that in the CA approach 400 cells are in state pedestrian and 
thus are red colored in $\Omega_{P E D}$. This is done by setting the distribution of red cells (i.e. the cells in the state pedestrian) randomly. Since one cell area is $0.16 \mathrm{~m}^{2}$ and the cell may be occupied at most by one pedestrian, we define the maximal density in the CA approach as $\rho_{\max }^{C A}:=1 / 0.16=6.25 \mathrm{ped} / \mathrm{m}^{2}$.

Analogously, as in Section 3.2.1, we use the random quantity $r \in[0,1)$ for the definition of the initial density distribution in the CA approach. We set $\rho_{C A}^{0}=2$ and use the following algorithm.

Algorithm 3: Initial pedestrian distribution setting.

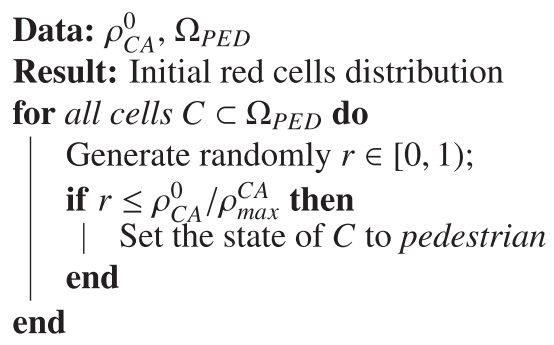

\subsection{CA density averaging}

To compare the density distribution in the CA approach and the numerical solution in the macroscopic model described in Section 2.2 we run repeatedly the CA simulations with the number of simulations NSIMUL $=100$. Then, we perform the following averaging of the CA pedestrian distribution density. If a cell $C \in \mathcal{T}_{h}$ was red colored $M$ times, we define the average $\widetilde{\rho}_{C}=M / N S I M U L$. We set $\widetilde{\rho}_{C}=0$ for $C \in \mathcal{T}_{w} \cup \mathcal{T}_{o}$. Finally, the quantity $\rho_{C}=\left(\frac{1}{9} \sum_{D \in N(C)} \tilde{\rho}_{D}\right) \rho_{\max }^{C A}$ is computed and drawn for comparison reasons with a macroscopic model.

In Figure 3 the initial condition (5.1) for the macroscopic model and for the CA are displayed, respectively.
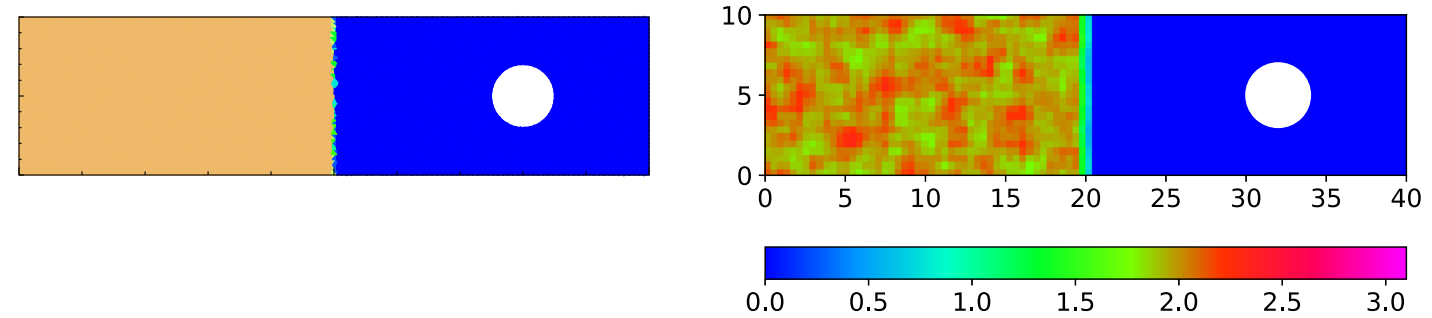

FIgURE 3. Initial condition for the macroscopic model (left) and averaged initial condition for CA approach (right).

\subsection{Setting the parameter $\boldsymbol{\beta}$ in $(3.2)$}

The probability of the move of the pedestrian in the proposed CA is given by formula (3.2). Here, the parameter $\beta$ has to be defined. We shall show the influence of the parameter $\beta$ on the numerical results obtained by CA for the CA from Figure 1 (right), i.e. for the evacuation problem with the initial condition (5.1).

In Figure 4 the pedestrian distribution is drawn for $\beta=5$ (left) and for $\beta=20$ (right) at time $t=10,15,20 \mathrm{~s}$.

As can be seen from Figure 4, the evacuation process is faster with an increased value of the parameter $\beta$. Since the time of evacuation $T_{\text {evac }}[\mathrm{s}]$ computed by $\mathrm{CA}$ is a significant quantity in the evacuation model, we compare the CA evacuation time with the evacuation time obtained via the finite volume method (FVM) from [19] on the same domain with the number of finite volumes $N E L E M=9056$. Here, the FVM evacuation time was $T_{\text {evac }}^{F V M}=36.5 \mathrm{~s}$. In Figure 5 (left) the number of pedestrians in the domain $\Omega$ against the time $t$ is shown. The curves show the decrease of number of pedestrians in the domain $\Omega$ during the evacuation process simulated by CA with various parameters $\beta$ in the probability matrix (3.2) and by FVM from [19]. The lowest 

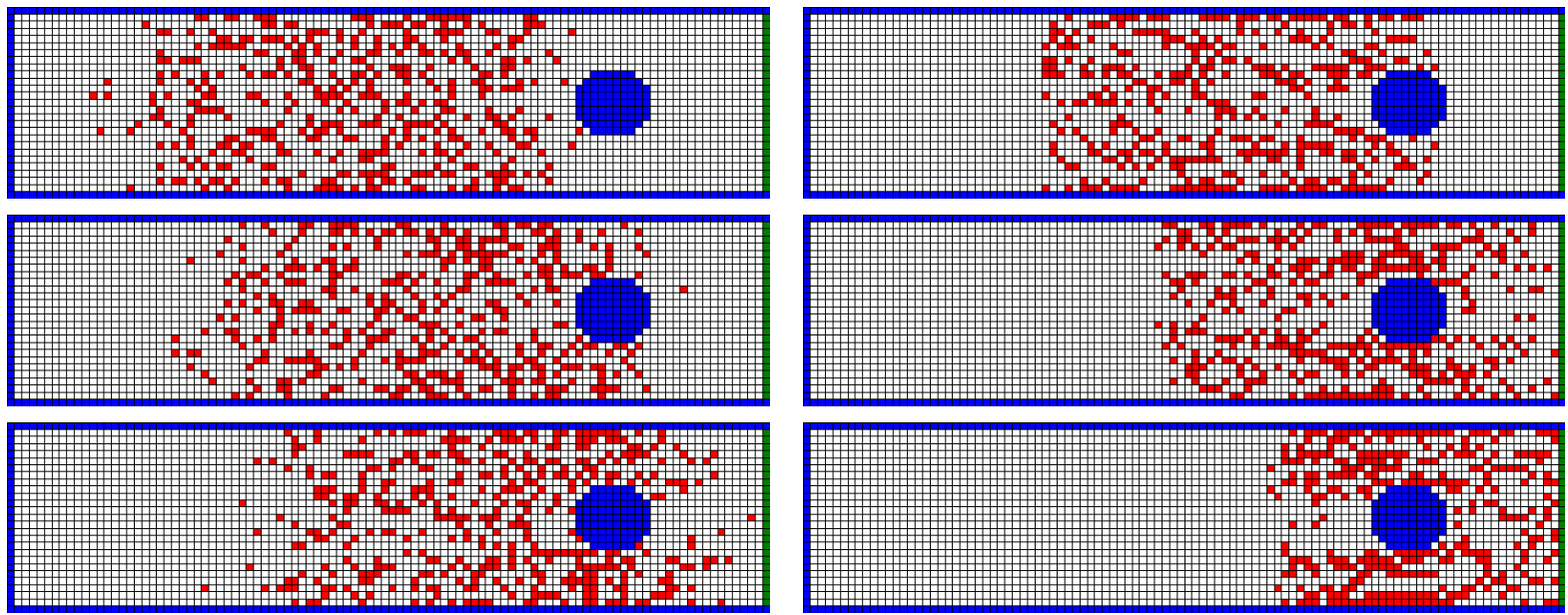

FIgURE 4. Domain with one obstacle, pedestrian distribution for $\beta=5$ (left) and $\beta=20$ (right) for time $t=10,15,20 \mathrm{~s}$.

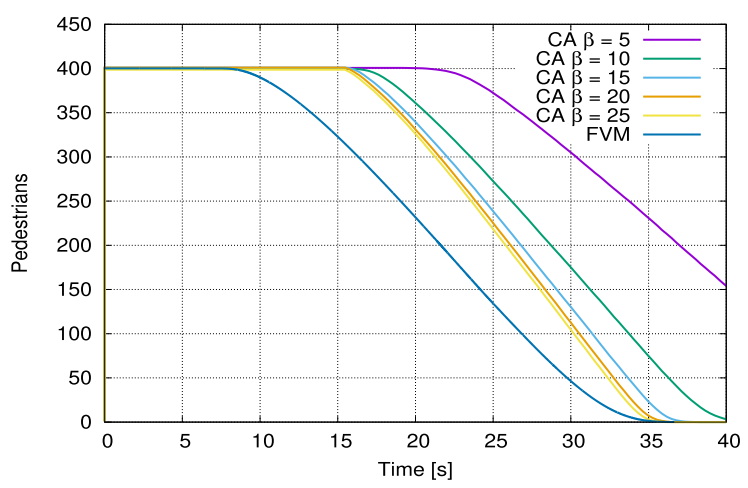

\begin{tabular}{|c|c|}
\hline$\beta$ & $T_{\text {evac }}[\mathrm{s}]$ \\
\hline 5 & 60.9 \\
10 & 40.1 \\
15 & 35.9 \\
20 & 34.7 \\
25 & 34.3 \\
\hline
\end{tabular}

Figure 5. Number of pedestrian in the domain during the evacuation process and the evacuation time $T_{\text {evac }}$.

(blue) curve corresponds to the results obtained in [19]. The CA evacuation time $T_{\text {evac }}$ corresponding to the various parameters $\beta$ is shown in the table in Figure 5 (right).

Based on these results the value $\beta=20$ is used in the following numerical examples.

\subsection{Evacuation of the domain with one circular obstacle}

We compare the results of the CA evacuation simulation for the domain with one circular obstacle from Figure 1 with the FV-DG method [9] (the Finite Volume Method (FV) for the space discretization and the Discontinuous Galerkin Method (DG) for the implicit time discretization). In this case, the system described in Section 2.2 is considered and the Eikonal equation is solved by the Dijkstra algorithm [23]. The initial condition (5.1) is used. In Figure 3 the initial distribution of the density for the FV-DG method and the averaged initial density for the CA at time $t=0$ is drawn, respectively. The averaging of the CA density described in Section 5.1 is further used in the CA simulations and the results at time $t=10,15,20 \mathrm{~s}$ are compared with those of FV-DG simulations from [9] in Figure 6. 

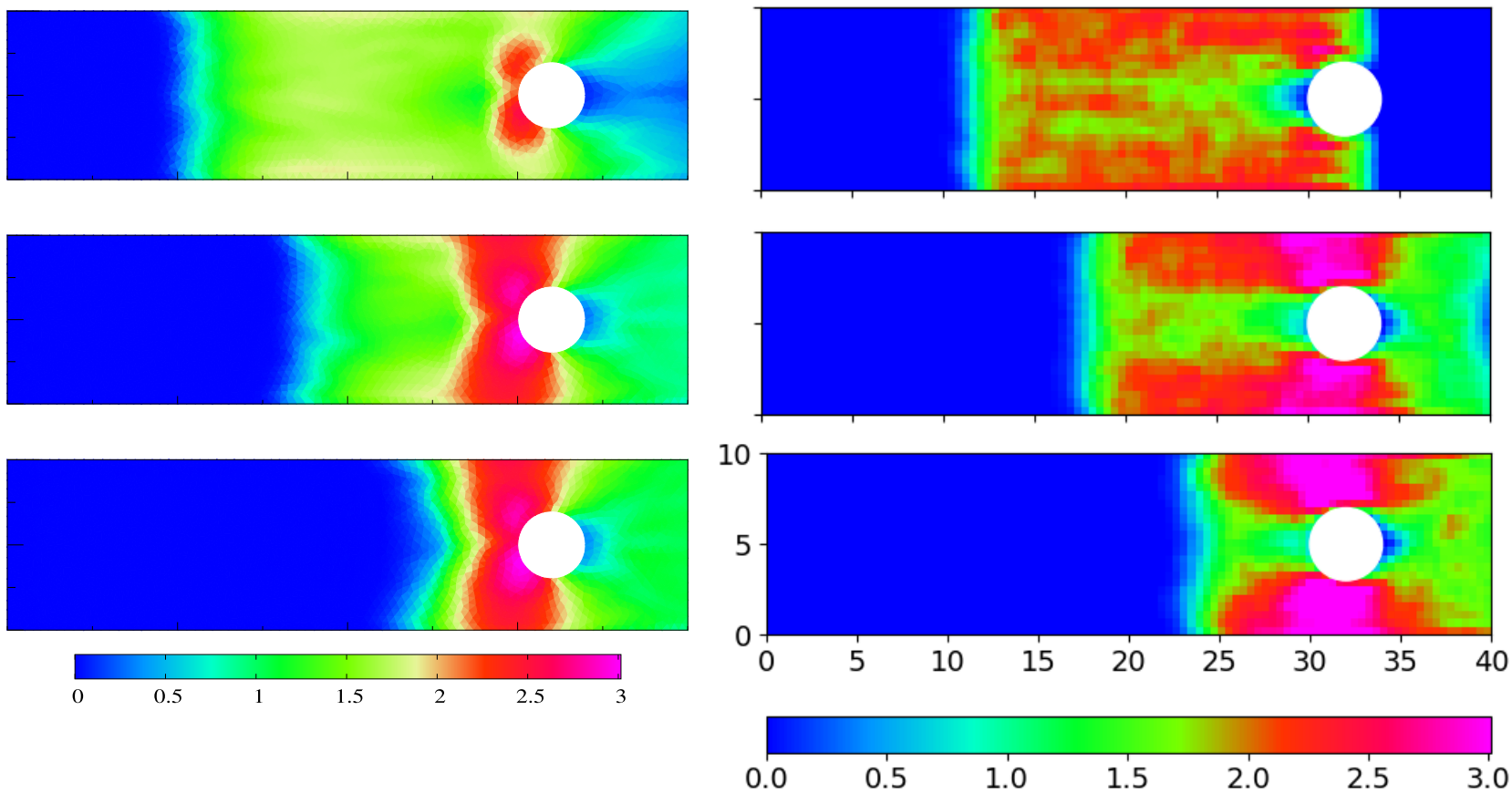

Figure 6. Density distribution over the domain with one circular obstacle at time $t=10,15,20 \mathrm{~s}$ obtained by the FV-DG method on the mesh with number of triangles $\# \mathcal{T}_{h}=$ 4627, Dijkstra algorithm (left) and $\mathrm{CA}$ algorithm with $\beta=20$ (right).

\subsection{Evacuation of the domain with three circular obstacles}

The results for the evacuation of the domain with more obstacles are presented in Figure 7. The same initial condition (5.1) as in the previous section is used. The FV-DG simulations from [9] and the CA simulations are mutually compared. The evacuated part of the room (blue colour in the left hand part of the domain) is in a good agreement for both methods.

For an intuitive validation of the CA model, the trajectories of some individual pedestrians are given in Figure 8.

\subsection{H-shape domain evacuation}

The domain $\Omega$ with two obstacles represented by symmetric re-entrant corners and with a non-symmetric position of the exit is considered.

$$
\Omega=\{(0,60) \times(0,25)\} \backslash\{\{[40,45] \times[0,7]\} \cup[40,45] \times[18,25]\}\},
$$

see Figure 9. The exit is defined

$$
\Gamma_{o}=\left\{x ; x_{1}=60, x_{2} \in(0,5)\right\}
$$

and the wall is $\Gamma_{w}=\partial \Omega \backslash \bar{\Gamma}_{o}$. We prescribe the initial condition

$$
\rho^{0}=\left\{\begin{array}{ll}
2 & x \in[0,20] \times[0,25], \\
0 & \text { elsewhere }
\end{array} \text { and } \boldsymbol{v}^{0}=\mathbf{0} .\right.
$$



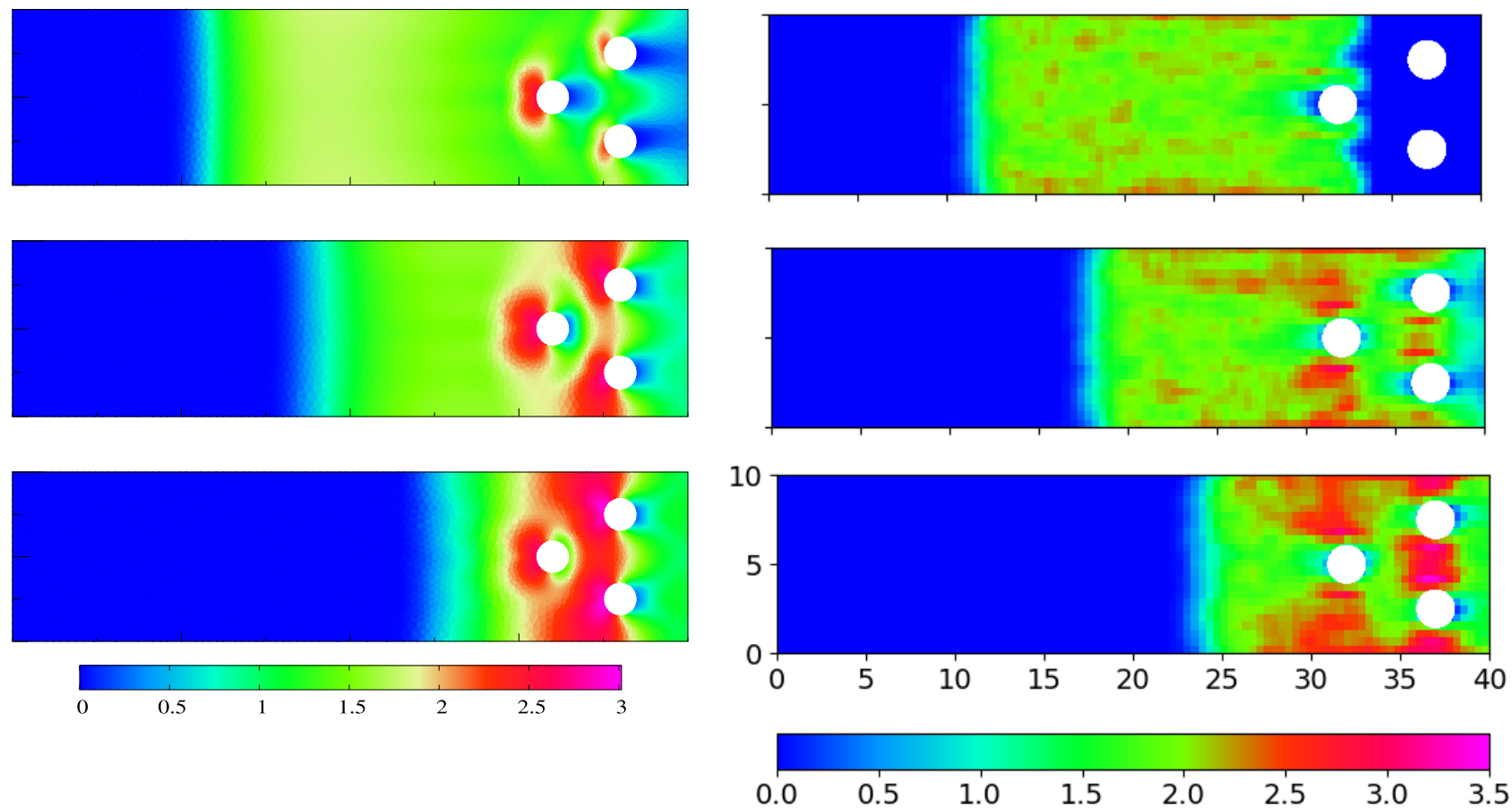

FiguRE 7. Domain with three circular obstacles at points $[32,5],[37,2.5]$ and $[37,7.5]$ with radius $R=1 \mathrm{~m}$, density distribution at time $t=10,15,20 \mathrm{~s}$ obtained by the FV-DG method on the mesh with the number of triangles $\# \mathcal{T}_{h}=7084$ with Bornemann-Rasch algorithm (left) and $\mathrm{CA}$ algorithm with $\beta=20$ (right).

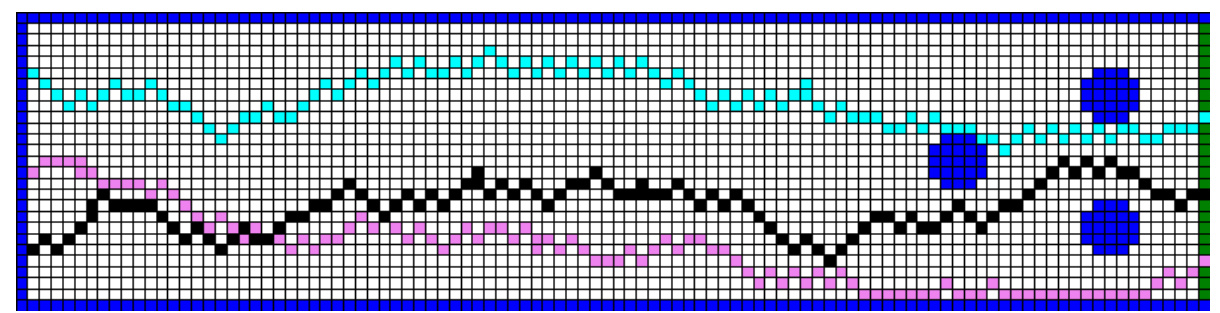

FiguRE 8. Domain with three obstacles - trajectories.

The initial condition in the CA setting is shown in Figure 9.

For PFE we carried out computations using Dijkstra's algorithm for the solution of the Eikonal equation and use our original results from [9] for the comparison with the CA approach. The PFE equations were solved on the mesh having 12742 triangles.

The density distributions achieved by FV-DG and CA method are depicted in Figures 10.

\subsection{T-shape domain evacuation}

To present the evacuation simulation results for the domain with multiple exits, we consider the so called T-shape domain which is depicted in Figure 11. 

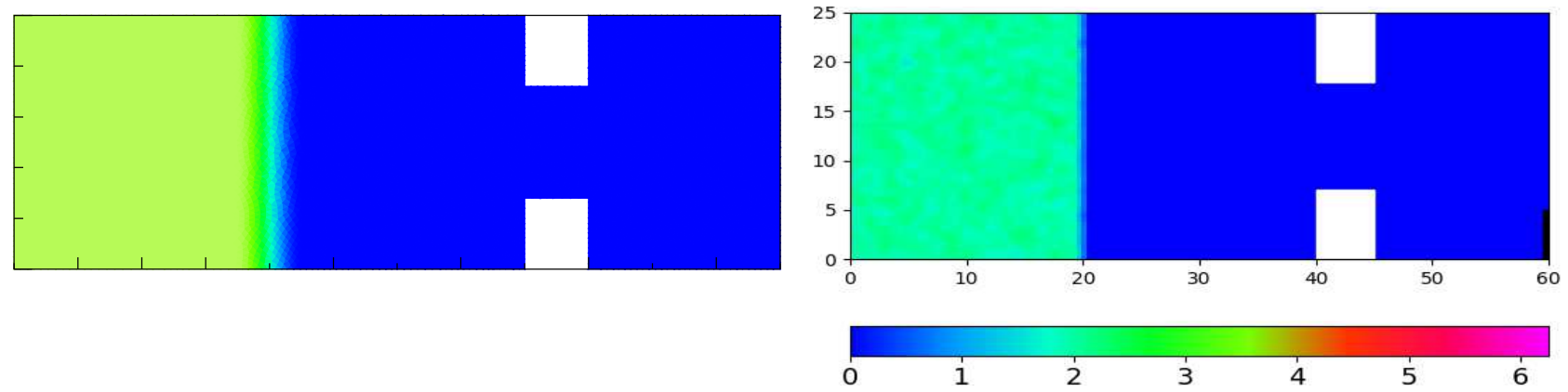

Figure 9. H-shape domain, initial pedestrian density distribution for FV-DG simulation (up) and for the CA simulation (down) for time $t=0 s$.
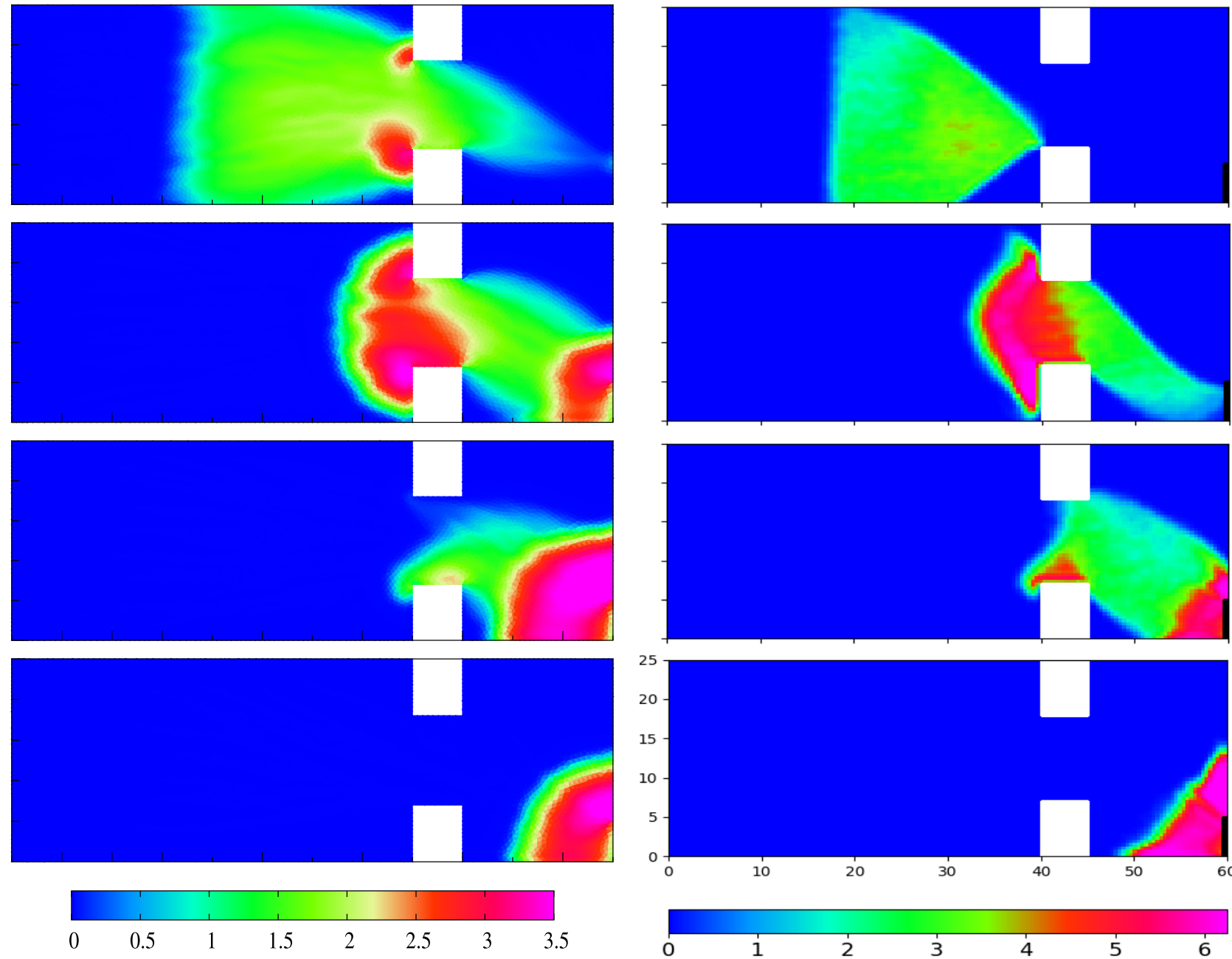

Figure 10. H-shape domain, pedestrian density distribution at time $t=15,30,45,60 \mathrm{~s}$ obtained by the FV-DG method (left) and CA (right). 

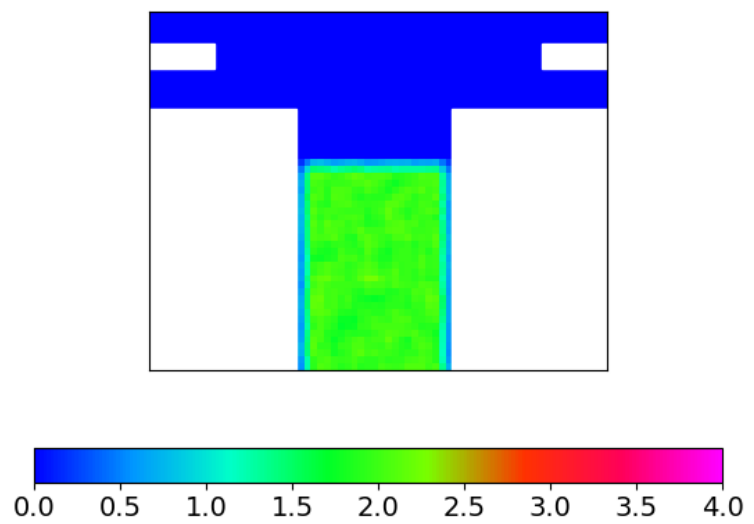

Figure 11. T-shape domain, CA initial pedestrian density distribution for time $t=0 \mathrm{~s}$.
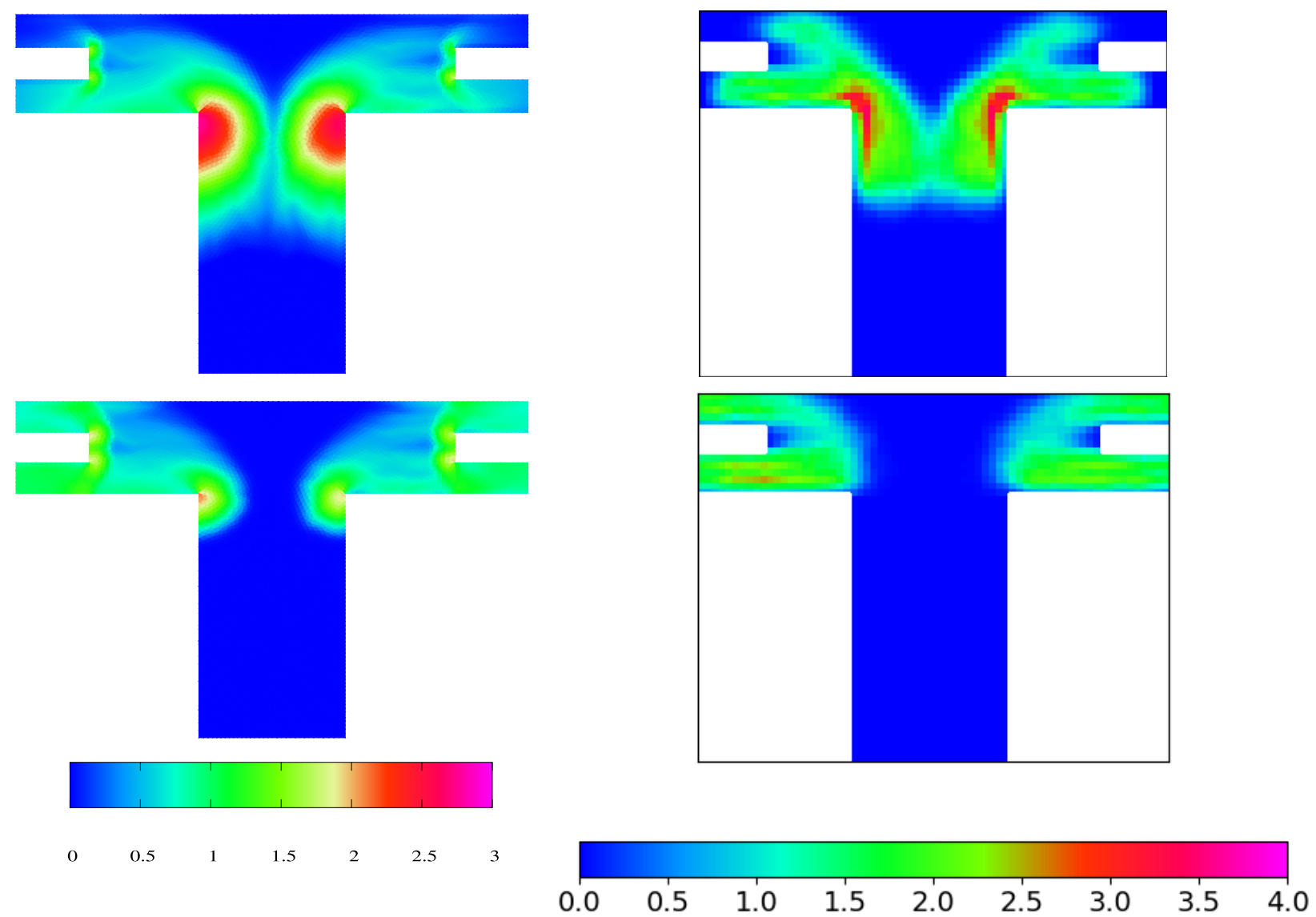

Figure 12. T-shape domain, pedestrian density distribution for time $t=9,15 \mathrm{~s}$, FV-DG method (left), CA (right).

The $x_{1}$-coordinates of the corners are $\{-14,-10,-4,4,10,14\}$ and the $x_{2}$-coordinates of the corners are $\{-16,0,2,4,6\}$. We consider the outflow $\Gamma_{o}$ on the vertical left- and right-hand sides of $\partial \Omega$, i.e.,

$$
\Gamma_{o}=\left\{x=\left(x_{1}, x_{2}\right) ; x_{1}=-14, x_{2} \in(0,2) \cup(4,6)\right\} \cup\left\{x=\left(x_{1}, x_{2}\right) ; x_{1}=14, x_{2} \in(0,2) \cup(4,6)\right\} .
$$



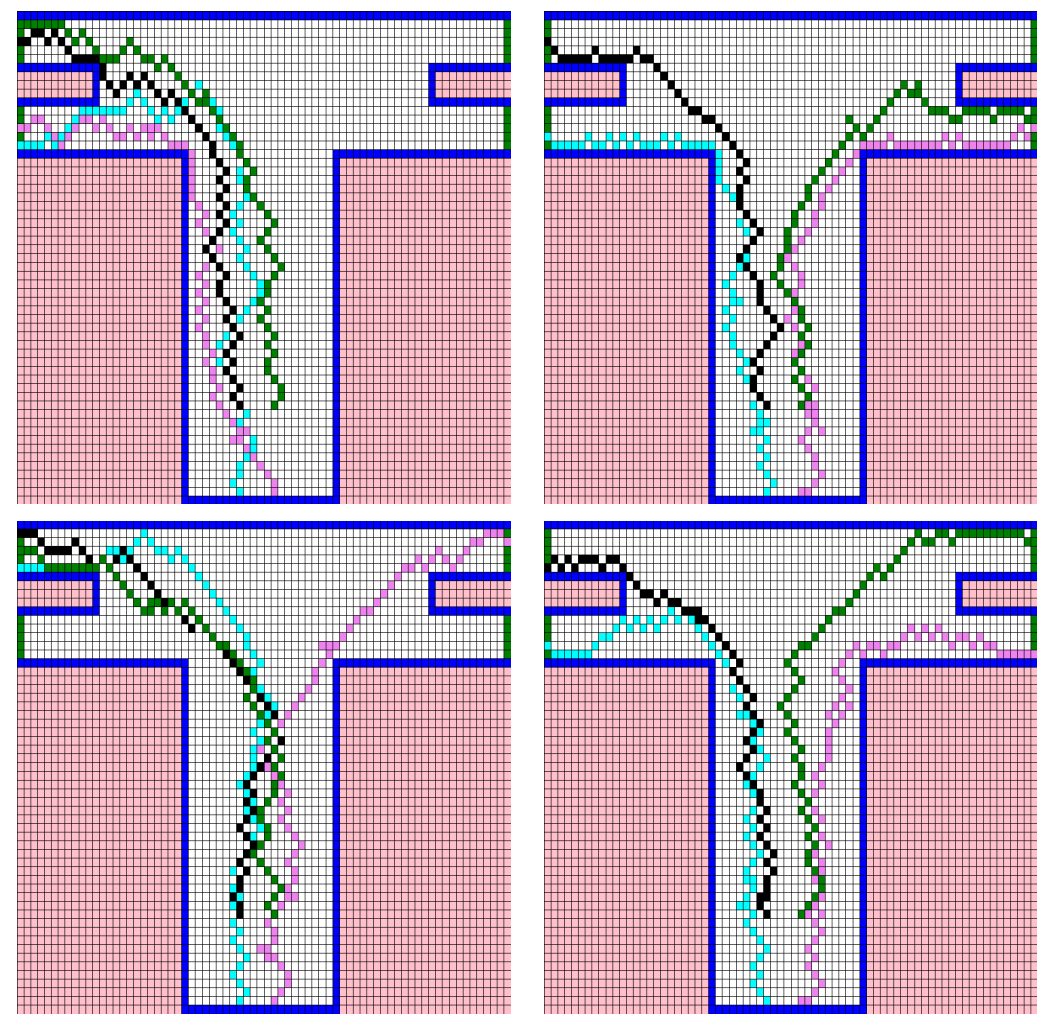

FiguRE 13. Trajectories of pedestrians at starting positions $[-1,-16]$ (blue trajectory), $[+1,-16]$ (pink trajectory), $[-1,-12]$ (black trajectory), $[+1,-12]$ (green trajectory).

Furthermore, the wall boundary is $\Gamma_{w}=\partial \Omega \backslash \bar{\Gamma}_{o}$. We prescribe the following initial condition:

$$
\rho^{0}=\left\{\begin{array}{ll}
2 & x \in[-4,4] \times[-16,-6], \\
0 & \text { elsewhere }
\end{array} \text { and } \boldsymbol{v}^{0}=\mathbf{0} .\right.
$$

The zero initial velocity here is intended for the FV-DG solution of PFE described in Section 2.2.

For PFE we carried out computations using Dijkstra's algorithm for the solution of the Eikonal equation and use our original results from [9] for the comparison with the CA approach. The PFE equations were solved on the mesh having 10848 triangles.

The density distributions achieved by FV-DG and CA method are depicted in Figures 12. The local increase of the pedestrian density near the corners in both approaches corresponds to an imagination of the real situation.

\subsubsection{Trajectories}

In the CA approach, by following a particular red cell (pedestrian) over the life time of the CA simulation, we can draw a trajectory of the pedestrian. In Figure 13 four pedestrians are considered at starting positions $[-1,-16]$ (blue trajectory), $[+1,-16]$ (pink trajectory), $[-1,-12]$ (black trajectory), $[+1,-12]$ (green trajectory). Clearly the randomness of trajectories due to the choice of the parameter $r$, see Section 3.2.1, can be observed. Nevertheless the trajectories are not in contradiction with an intuitive expectation. It is worth noting that due to the local increase of the pedestrian density near the corners, the trajectories may end at an exit more distant then the nearest exit to their initial positions. 


\section{Conclusion}

A new cellular automaton for the pedestrian evacuation simulation has been proposed and numerically tested. The benefits of this approach are twofold. The FFCA model from [5] is futher developed by incorporating the Eikonal equation into the probability matrix $P^{C}$. The idea of the construction of the probability matrix from [5] is taken into account and the parameter dependent formula (3.2) is proposed. The role of the parameter $\beta$ in (3.2) is explained, and $\beta=20$ is used in a series of computations.

Furthermore, we tried to show a comparison of the connection between two different worlds for modeling of pedestrian evacuation, the world of partial differential equations and the world of cellular automata. Although the conceptual structure of the two worlds is very different and the PFE model uses a very deep theory of the discontinuous Galerkin method and error estimates, the CA approach provides an idea of pedestrian behavior that is in good agreement with the numerical results using PFE.

The proposed CA model can be further developed. The diffusion equation will be the first choice to enrich the model. The interconnection of CA and neural networks can also be considered.

Acknowledgements. The research was supported by the grant No. UJEP-IGA-TC-2019-53-02-2 of IGA UJEP. The authors acknowledge this support.

\section{REFERENCES}

[1] S. Bandini, G. Mauri and R. Serra, Cellular automata: From a theoretical parallel computational model to its application to complex systems. Parallel Comput. 27 (2001) 539-553.

[2] N. Bellomo and Ch. Dogbé, On the modelling crowd dynamics from scaling to hyperbolic macroscopic models. Math. Models Methods Appl. Sci. 18 (2008) 1317-1345.

[3] N. Bellomo, A. Marasco and A. Romano, From the modelling of driver's behavior to hydrodynamics models and problems of traffic flow. Nonlinear Anal. RWA 3 (2002) 339-363.

[4] S. Buchmueller and U. Weidmann, Parameters of pedestrians, in: Pedestrian Traffic and Walking Facilities. Technical report, Schriftenreihe des IVT, ETH Zurich (2006).

[5] C. Burstedde, K. Klauck, A. Schadschneider and J. Zittartz, Simulation of pedestrian dynamics using a two-dimensional cellular automaton. Physica A 295 (2001) 507-525.

[6] E. Cristiani, B. Piccoli and A. Tosin, Multiscale Modeling of Pedestrian Dynamics. Springer International Publishing (2014).

[7] F. Dietrich and G. Köster, Gradient navigation model for pedestrian dynamics. Phys. Rev. E 89 (2014) 062801.

[8] C. Dogbe, On the modelling of crowd dynamics by generalized kinetic models. J. Math. Anal. Appl. 387 (2012) $512-532$.

[9] V. Dolejší, J. Felcman and P. Kubera, FV-DG method for the Pedestrian flow problem. Comput, Fluids 183 (2019) 1-15.

[10] M.H. Dridi, Simulation of high density pedestrian flow: Microscopic model. Open J. Modell. Simul. 3 (2015) 81-95.

[11] M. Feistauer, J. Felcman and I. Straškraba, Mathematical and computational methods for compressible flow. Clarendon Press (2003).

[12] J. Felcman and P. Kubera, Eikonal equation based cellular automaton for a pedestrian evacuation problem. In T. E. Simos and Ch. Tsitouras, editors, ICNAAM 2019 AIP Conference Proceedings. American Institute of Physics (2019) 1-4.

[13] Z. Fu, X. Zhan, L. Luo, A. Schadschneider and J. Chen, Modeling fatigue of ascending stair evacuation with modified fine discrete floor field cellular automata. Phys. Lett. A 383 (2019) 1897-1906.

[14] J. Gao, J. He and J. Gong, A simplified method to provide evacuation guidance in a multi-exit building under emergency. Physica A $\mathbf{5 4 5}$ (2020) 123554.

[15] D. Helbing and P. Molnár, Social force model for pedestrian dynamics. Phys. Rev. E 51 (1995) 4282-4286.

[16] Y. Jiang, B. Chen, X. Li and Z. Ding, Dynamic navigation field in the social force model for pedestrian evacuation. Appl. Math. Modell. 80 (2020) 815-826.

[17] Y.Q. Jiang, P. Zhang, S.C. Wong and R.X. Liu, A higher-order macroscopic model for pedestrian flows. Physica A 389 (2010) $4623-4635$.

[18] A. Kirchner and A. Schadschneider, Simulation of evacuation processes using a bionics-inspired cellular automaton model for pedestrian dynamics. Physica A 312 (2002) 260-276.

[19] P. Kubera and J. Felcman, On a numerical flux for the pedestrian flow equations. JAMSI 11 (2015) 79-96.

[20] Y. Li, M. Chen, Z. Dou, X. Zheng, Y. Cheng and A. Mebarki, A review of cellular automata models for crowd evacuation. Physica A $\mathbf{5 2 6}$ (2019) 120752.

[21] R. Liu, Z. Fu, A. Schadschneider, Q. Wen, J. Chen and S. Liu, Modeling the effect of visibility on upstairs crowd evacuation by a stochastic ffca model with finer discretization. Physica A 531 (2019) 121723.

[22] H.J. Payne, Models of freeway traffic and control. Simulation Councils, Incorporated (1971).

[23] T. Petrášová, Application of the Dijkstra's algorithm in the pedestrian flow problem. Bc thesis, Charles University in Prague (2016). 
[24] K. Rendón Rozo, J. Arellana, A. Santander-Mercado and M. Jubiz-Diaz, Modelling building emergency evacuation plans considering the dynamic behaviour of pedestrians using agent-based simulation. Saf. Sci. 113 (2019) 276-284.

[25] A. Seyfried, B. Steffen and T. Lippert, Basics of modelling the pedestrian flow. Physica A 368 (2006) $232-238$.

[26] L. Tan, M. Hu and H. Lin, Agent-based simulation of building evacuation: combining human behavior with predictable spatial accessibility in a fire emergency. Inf. Sci. 295 (2015) 53-66.

[27] M. Twarogowska, P. Goatin and R. Duvigneau, Macroscopic modeling and simulation of room evacuation. Appl. Math. Modell. (2014) 5781-5795.

[28] G.B. Whitham, Linear and nonlinear waves. Pure and applied mathematics, Wiley (1974).

[29] P. Zhang, X.-X. Jian, S.C. Wong and K. Choi, Potential field cellular automata model for pedestrian flow. Phys. Rev. E 85 (2012) 021119.

[30] Hk. Zhao, A fast sweeping method for Eikonal equations. Math. Comput. 74 (2005) 603-627. 